\title{
Secondary Mitral Regurgitation in Heart Failure with Reduced or Preserved Left Ventricular Ejection Fraction
}

\author{
Pierre Vladimir Ennezat ${ }^{\mathrm{a}} \quad$ Sylvestre Maréchaux ${ }^{\mathrm{b}} \quad$ Philippe Pibarot $^{\mathrm{c}}$ \\ Thierry H. Le Jemtel ${ }^{d}$

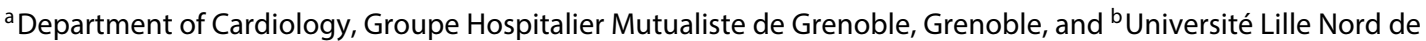 \\ France, GCS - Groupement des Hôpitaux de l'Institut Catholique de Lille/Faculté Libre de Médecine, Université \\ Catholique de Lille, Lille, France; 'Institut Universitaire de Cardiologie et de Pneumologie de Québec/Quebec Heart

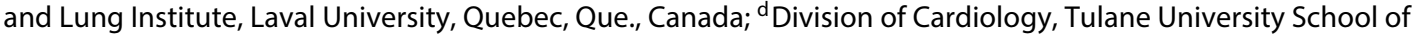 \\ Medicine, New Orleans, La., USA
}

\section{Key Words}

Secondary mitral regurgitation $\cdot$ Heart failure $\cdot$ Diastolic dysfunction · Echocardiography

\section{Abstract \\ Secondary mitral regurgitation (MR) has been extensively studied in heart failure due to reduced ejection fraction. In contrast, the occurrence and the pathogenesis of secondary $M R$ are much less known in heart failure with preserved ejec- tion fraction (HFpEF). The present review aimed at describ- ing this common but ignored feature of HFpEF.}

Copyright $\odot 2013$ S. Karger AG, Basel

As in patients with heart failure (HF) and reduced left ventricular (LV) ejection fraction ( $\mathrm{HFrEF})$, patients with $\mathrm{HF}$ and preserved LV ejection fraction (HFpEF) may develop secondary (or functional) mitral regurgitation (MR) $[1,2]$. The aim of this article is to review the mechanisms and the clinical significance of secondary MR in HFrEF and HFpEF patients.

\section{KARGER}

E-Mail karger@karger.com

www.karger.com/crd

\section{Secondary MR in HFrEF}

Independently of the etiology of HFrEF and its underlying mechanisms, secondary MR portends a poor clinical outcome [3]. Early after myocardial infarction, moderate or severe MR was found to be independently associated with an increased risk of HF and death (relative risk 3.44 and 1.55, respectively) in a large communitybased cohort [4]. The downward spiral of MR that begets MR affects the progression of HFrEF [5]. Secondary MR in HFrEF reflects primarily the severity of LV dysfunction and is not related to structural alterations of the mitral valvular apparatus. Nevertheless, mitral valves from hearts collected at the time of cardiac transplantation are biochemically different from those from normal hearts. Mitral valve remodeling resulting from increased deoxyribonucleic acid, glycosaminoglycan and collagen concentration develops in patients with LV systolic dysfunction [6]. Mitral valve area increases over time as the left ventricle remodels in an experimental model of inferior myocardial infarction [7]. This suggests that secondary $\mathrm{MR}$ in these HF patients may not be purely functional. 
Fig. 1. Mechanisms leading to secondary

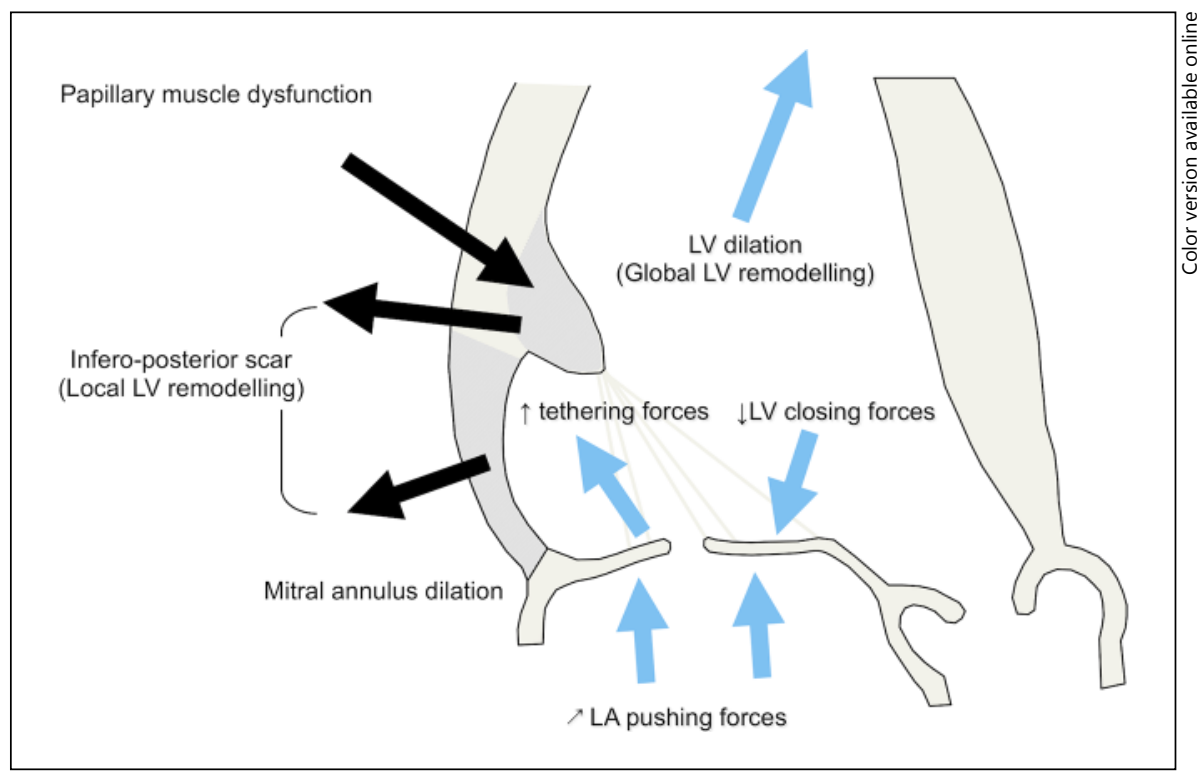
MR in HFrEF.

Apical displacement of the papillary muscles due to eccentric global and local LV remodeling and segmental wall motion abnormalities associated with papillary muscle dysfunction increase the tethering forces acting on the mitral valve. Reduced LV contractility, LV dyssynchrony and reduced mitral annular contractility decrease the LV systolic pressure that in turn results in reduced mitral valve closing forces. Importantly, the hallmark of secondary MR in HFrEF is the formation of mitral valve tenting (a more apical position of the leaflets and their coaptation point during the systolic phase) that may be asymmetric if the tethering forces predominate on the posterior mitral leaflet [8]. It is currently accepted that the mitral valve tenting results from an imbalance between these increased tethering forces and reduced closing forces $[9,10]$.

The concept that heightened left atrial (LA) pressure may induce mitral valve tenting, and thereby secondary MR has recently been reported. Park et al. [11] showed in 144 patients with dilated cardiomyopathy that the severity of secondary MR most significantly correlates with the mitral valvular tenting area, which is in turn more closely related to mitral annular size and diastolic dysfunction severity than to LV volume. Conversely, the E/e' ratio, a noninvasive estimate of LV filling pressures, is independently predicted by secondary MR severity irrespective of age, gender and LV ejection fraction in a large cohort of patients with coronary artery disease [12]. Even mild MR was associated with an increased E/e' ratio [12]. Our group demonstrated that in addition to the posterior pap-

Secondary Mitral Regurgitation in Heart Failure illary muscle-intervalvular fibrosa distance, a key parameter of local LV remodeling, a higher E/e' ratio, is an independent predictor of the systolic mitral tenting area and ensuing MR in patients with HFrEF [13]. Hence, diastolic dysfunction and thereby increased LA pressure appear to be important contributing factors for secondary MR development in patients with LV systolic dysfunction through the increased mitral valve tenting area owing to increased LA size and LA 'pushing' forces. Figure 1 summarizes the mechanisms that promote secondary MR in patients with HFrEF.

Previous studies have demonstrated that secondary $\mathrm{MR}$ is in part responsible for pulmonary hypertension in $\mathrm{HFrEF}$, a major predictor of poor outcome [14]. In a recent study, Miller et al. [15] confirmed that, irrespective of the severity of diastolic dysfunction (E/e' ratio $\geq 15$, odds ratio: $3.31, \mathrm{p}<0.001$; deceleration time $\leq 150 \mathrm{~ms}$, odds ratio: 3.8, $\mathrm{p}<0.001$ ), secondary MR [effective regurgitant orifice area (EROA) $\geq 20 \mathrm{~mm}^{2}$, odds ratio: $3.8, \mathrm{p}<0.001$ ] was a powerful predictor of pulmonary hypertension.

Secondary MR depends heavily on loading conditions (therapeutic compliance and daily salt intake) and ventricular performance. The volume of mitral regurgitant flow is determined hydraulically by two factors: magnitude and duration of the systolic pressure gradient across the valve and size of the EROA. In acutely decompensated HF, both diuretic and venodilation with nitroglycerin lower pulmonary venous pressure, resulting in a fall in LA pressure that is greater than the reduction in LV systolic pressure, thereby increasing the gradient across the mitral 
valve and reducing the size of the EROA [16]. The reduction in LV size and thereby in annular distension also accounts for a reduction in the EROA, especially in patients with HFrEF who are receiving ACE inhibition and $\beta$-adrenergic blockade therapy $[17,18]$. Altogether, a decrease in regurgitant flow is associated with an improved forward stroke volume and thereby end-organ perfusion. Besides increased loading conditions, active myocardial ischemia contributes to worsening secondary MR, while medical and nonpharmacological treatments including revascularization and cardiac resynchronization therapy reduce the amount of MR [19-24]. A major feature of secondary MR in HFrEF is its dynamic nature with exercise. A large exercise-induced increase in secondary MR $\left(>13 \mathrm{~mm}^{2}\right)$ has been linked to poor outcome in previous reports involving patients with LV systolic dysfunction due to coronary artery disease [25], while the role of dynamic exercise MR has been found to be less important in nonischemic and revascularized ischemic HFrEF patients [26]. Patients with coronary artery disease and a recent history of pulmonary edema experience a greater increase in MR during exercise suggesting the importance of secondary ischemic MR in acute decompensation of HFrEF [27].

\section{Secondary MR in HFpEF}

Patients with HFpEF have as poor a prognosis as those with HFrEF, and HFpEF is responsible for as many hospitalizations as HFrEF is $[28,29]$. Although it imposes a huge burden on the healthcare system, HFpEF remains a poorly understood clinical entity that has no evidencebased therapy as illustrated by consistently negative clinical trials $[30,31]$. A potential explanation for the failed cardiovascular therapeutic approach of HFpEF may be that a majority of the HFpEF patients (60\%) died of noncardiovascular causes, while only $36 \%$ of the HFrEF patients died of noncardiovascular causes [29]. The phenotype of HFpEF is well known: elderly women with hypertension, diabetes and obesity as comorbid conditions [32]. However, its pathogenesis is multifaceted. Investigators have highlighted the role of combined ventricular and arterial stiffening, subtle longitudinal and torsional LV systolic dysfunction, chronotropic incompetence and renal impairment in HFpEF pathogenesis [32, 33]. Overall HFpEF is a less homogenous condition than HFrEF is. Comorbidities appear to play a greater role in the pathogenesis of HFpEF than they do in HFrEF [34-37]. In such contexts it is not surprising that a lot less is known regarding the potential role of secondary MR in HFpEF.
Symptomatic patients with HFpEF may present with MR without typical lesions of the mitral valve apparatus commonly observed in organic MR [1]. While mitral valve remodeling has been demonstrated in patients with HFrEF, no pathology data exist in patients with HFpEF. Nevertheless, similar as in patients with HFrEF, the mitral valve may or may not be strictly normal in HFpEF patients with secondary MR owing to the age of the patient population. An increased leaflet thickness especially at the basal third of the valve (greater stress localization) is noticed in these older patients. Mitral valve leaflets become less translucent with age, and adipocytes and lipid deposits accumulate within the leaflet tissue. Nodular thickening can often be seen along the lines of closure on both leaflets promoting a degree of leaflet miscoaptation [38]. It is important to note that due to aging, calcifications of the mitral annulus are a common occurrence in HFpEF [39].

Secondary MR is found in a significant proportion of patients with HFpEF [2]. Mitral regurgitant volume is usually in the range of that observed in mild MR owing to a small LV cavity [2]. It is of note that due to unreliable semiquantitative assessment by Doppler color flow mapping (exquisite sensitivity of the color Doppler jet area to driving pressure, chamber constraint, gain settings, LA size and compliance) MR may appear hemodynamically severe upon admission and some patients with HFpEF are referred for mitral valve surgery despite the absence of overt lesions of the mitral valve apparatus [40,41]. Combined increased LA pressure and atrial fibrillation (which occurs frequently in HFpEF) produce enlarged LA volume, an important feature of HFpEF [42]. LA size affects mitral annular size independent of LV dimensions [43]. Mitral annulus dilatation as a result of LA enlargement promotes the development of secondary MR [43]. It is interesting to note that the concept of 'atrial functional MR' has recently been associated with hypertensive and older patients with atrial fibrillation who develop MR. Atrial fibrillation induces mitral annular distortion and dysfunction and loss of atrial synchrony both contributing to MR. Conversely MR is improved after successful atrial fibrillation ablation [44]. Concentric LV remodeling, a common feature of HFpEF, rules out that increased tethering forces play a major role in the development of MR in these HFpEF patients. Tethering forces may nevertheless play a role in the subgroup of HFpEF patients with eccentric LV remodeling [13]. Whether dysfunctional subendocardial longitudinal fibers (as assessed by the reduced diastolic and systolic mitral annular velocities e' and s') lower closing forces and thereby play a role in the pathogenesis of secondary MR in HFpEF has not been investigated. LV 


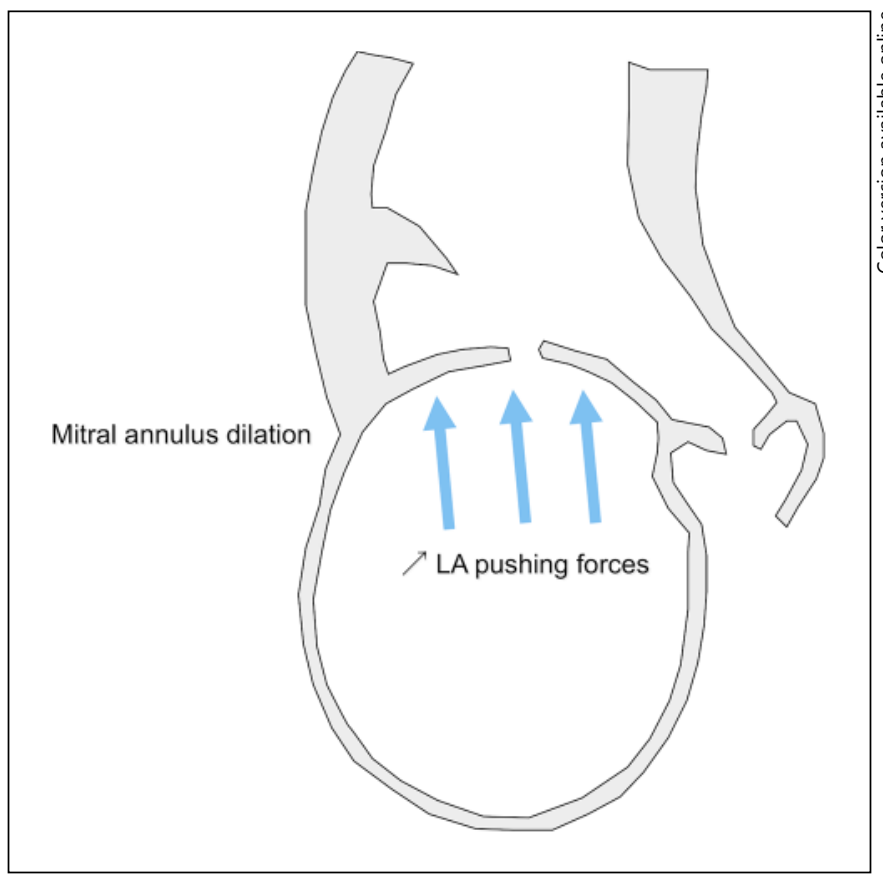

Fig. 2. Mechanisms leading to secondary MR in HFpEF.

dyssynchrony has been reported in HFpEF patients with narrow QRS [45]. LV dyssynchrony is load dependent [46, 47] and associated with discoordinated papillary muscle contraction and impaired LV performance. Although the relationship between LV dyssynchrony and MR has been extensively studied in HFrEF, its role remains to be established in HFpEF. Mitral valve tenting and miscoaptation are likely consequences of increased LV filling pressure and of the resulting increased LA pressure that opposes mitral leaflet closure. Of note the E/e' ratio is independently associated with mitral valve tenting after adjustment for LV remodeling (interpapillary muscle distance) in the subset of patients with HFpEF [13]. Consistently, for a similar degree of $L V$ remodeling or function, the increase in LA pressure determines mitral valve tenting [13]. Discrete mitral annular calcifications may restrict leaflet motion and act as a substrate to secondary MR. Figure 2 summarizes the mechanisms that promote secondary MR in patients with HFpEF.

Figure 3 and video 1 (online suppl. video 1; for all online suppl. material, see www.karger.com/doi/10.1159/ 000350356) show the case of a 65-year-old hypertensive patient presenting with acute HF with secondary MR, increased LV filling pressure and normal LV ejection fraction. Unloading therapy was associated with a decrease in LV preload mirrored by a major decrease in MR [1]. Sim-

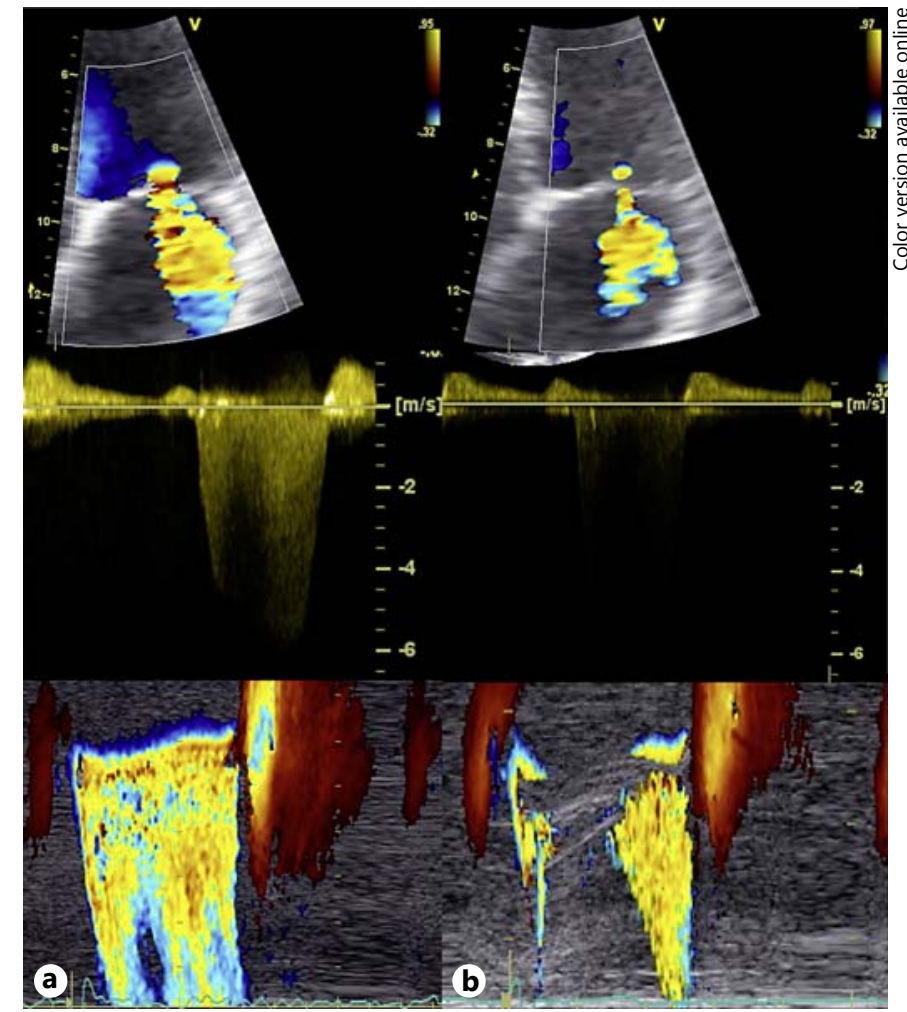

Fig. 3. Quantification of the degree of functional MR in a patient with HFpEF before (a) and after (b) unloading therapy. The reduction in the severity of MR is obvious. Note the increase in continuous wave Doppler signal and in proximal flow convergence radius in early and late systole as commonly encountered in LV systolic dysfunction [1, with permission].

ilar to patients with LV systolic dysfunction and secondary $\mathrm{MR}$, analysis of the proximal flow convergence often displays peaks in the early and late systole and a midsystolic decrease. Midsystolic decreases in the regurgitant orifice area mirror increases in transmitral pressure acting to close the valve (fig. 3) [48].

Although coronary artery disease is less prevalent in HFpEF than in HFrEF, myocardial ischemic disease should definitely be excluded as a cause of dynamic or transient MR in HFpEF patients. Myocardial infarction of small size (particularly in the posterior wall) or ischemia cause LV remodeling, subendocardial dysfunction, papillary muscles and segmental wall motion abnormalities and dyssynchrony, all factors known to facilitate acute MR and HF [49]. Nevertheless, coronary artery disease is commonly associated with mitral annular calcifications [50].

As previously found in HFrEF, secondary MR has been linked to pulmonary hypertension in HFpEF irrespective of the severity of LV diastolic dysfunction [2]. 
Table 1. Features of secondary MR in HFrEF and HFpEF patients

\begin{tabular}{lll}
\hline & HFrEF & HFpEF \\
\hline Eccentric global LV remodeling & +++ & + \\
Local LV remodeling & ++++ & + \\
Mitral annular dilation and dysfunction & +++ & +++ \\
LV contractility & ++ & $?$ \\
LV dyssynchrony & +++ & $?$ \\
LA pressure & + & +++ \\
Atrial fibrillation & + (LV systolic dysfunction- and age-related) & ++ \\
Structural mitral valve changes & +++ & $++($ age-related) \\
Dynamic changes with exercise & needs to be considered & +++ \\
Active myocardial ischemia & +++ & needs to be considered \\
Linked to pulmonary hypertension & +++ & +++ \\
Poor outcome & $?$ \\
\hline
\end{tabular}

$+++=$ Highly related to secondary MR; ++ = moderately related to secondary MR; + = mildly related to secondary MR; ? = unknown.

Indeed, MR reduces forward LV outflow and in turn increases LV filling pressure and pulmonary venous hypertension. Pulmonary pressures fluctuate in patients with HFpEF with secondary MR of varying severity. Overall, pulmonary hypertension is frequent in patients with HF$\mathrm{pEF}$ and portends a poor prognosis as in patients with HFrEF [51]. However, the severity of pulmonary venous hypertension (combined role of LV diastolic dysfunction and MR) does not fully account for the severity of pulmonary hypertension in HFpEF $[2,51]$. Pulmonary vascular resistances may increase out of proportion to the rise in pulmonary venous pressure in these aged and hypertensive patients who exhibit increased vascular stiffness.

Two-dimensional Doppler echocardiography examination during exercise reveals the dynamic nature of secondary MR in HFpEF as in HFrEF patients (fig. 4) [52, 53]. Once myocardial ischemia is excluded $[54,55]$, an acute increase in LV diastolic stiffness and thereby in LV filling pressure during exercise (as estimated from the E/e' ratio [56]) may increase or produce secondary MR owing to mitral leaflet miscoaptation [51]. Several investigators have noted an abnormal LV response to exercise that may exacerbate MR [53, 57-60]. Whether LV dyssynchrony plays a role in the worsening of secondary MR during exercise is under investigation [61].

The prognostic importance of secondary nonischemic MR in HFpEF is currently unknown. As in patients with HFrEF, unloading therapies that improve signs and symptoms of HFpEF patients usually reduce or even suppress MR [1]. Worsening renal function may limit the efficacy of unloading therapies owing to an excessive rise in cardiac preload. Eventually mitral valve replacement may be considered in HFpEF patients with persistent MR or severe inducible exercise MR. However, the outcome of mitral surgical procedures is unproven in this elderly population where comorbid conditions play a major role in prognosis [34]. Table 1 shows the comparison of secondary MR features between HFrEF and HFpEF patients.

\section{Clinical Implications of Loading Conditions: Importance in Secondary MR}

Patients with acutely decompensated HF due to either HFrEF or HFpEF should be assessed for MR after stabilization. A comparison between echocardiographic findings at the time of acute decompensation and after stabilization may help to identify MR mechanisms: worsening of LV diastolic dysfunction and/or LV systolic dysfunction? Substantial concomitant decreases in pulmonary pressures are also observed after unloading therapy.

\section{Conclusion}

Secondary MR occurs in patients with HFpEF as it does in those with HFrEF. Whether secondary MR is a bystander or an actor in HF remains a source of debate. Secondary MR associated with HFpEF or HFrEF appears 
Fig. 4. An exercise-induced increase in MR that is trivial at rest in a 77-year-old hypertensive patient with HFpEF. The increase in mitral EROA (trivial up to $9 \mathrm{~mm}^{2}$ ) is paralleled by an increase in the mitral tenting area $\left(2-3.2 \mathrm{~cm}^{2}\right)$.
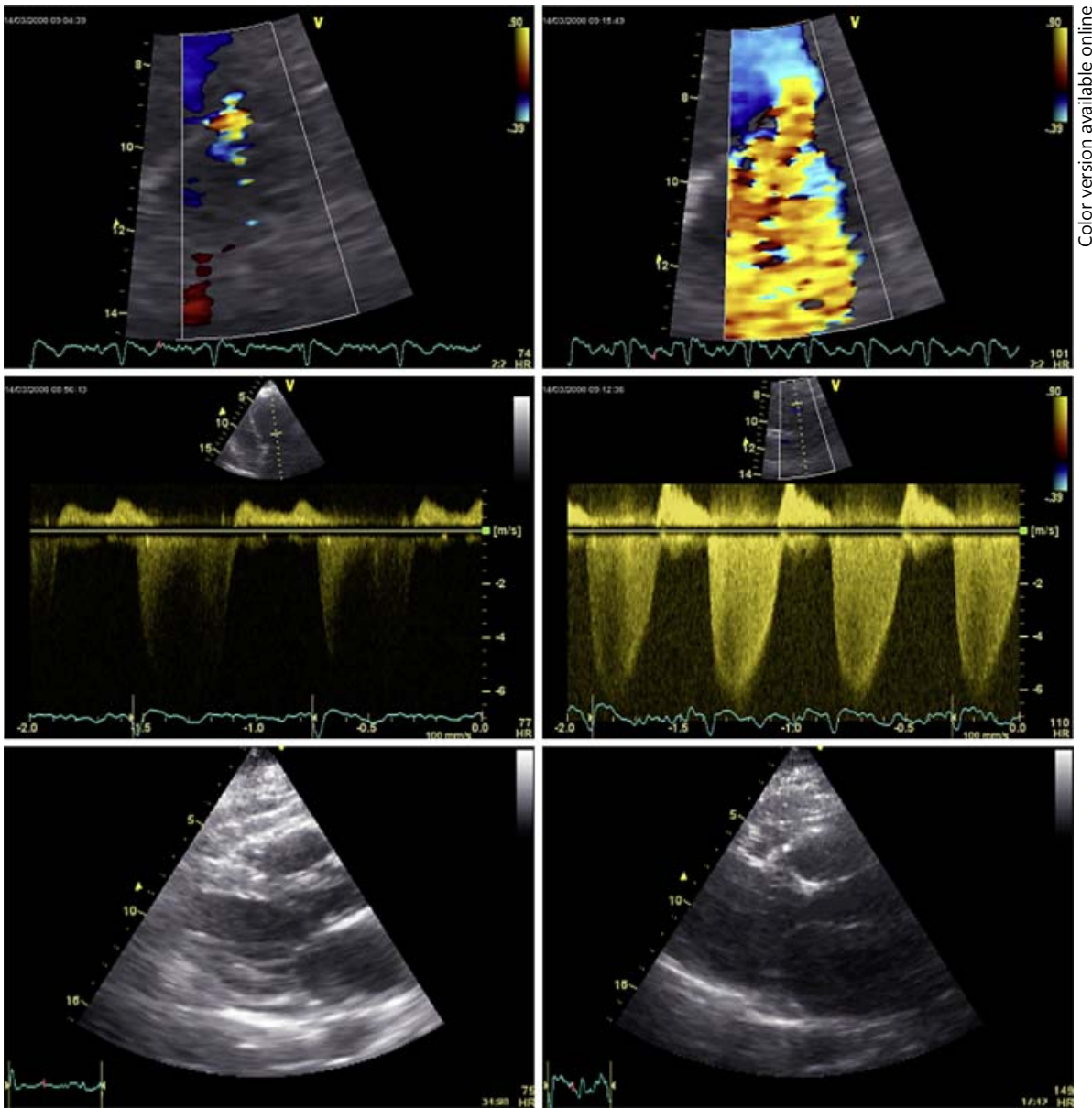

to be highly dependent on cardiac loading conditions. Careful assessment of the mitral valve apparatus and subapparatus is needed to exclude organic lesions including focal prolapse, rheumatic or drug-induced lesions. Ischemic disease should be clearly ruled out as a cause of dynamic or transient MR in HF patients. Secondary $\mathrm{MR}$ is an independent determinant of pulmonary hypertension in both patients with HFpEF or HFrEF. Hence, the finding even of a mild secondary MR with HFpEF is likely to be viewed as an HF diagnostic criterion in patients with suspected HF despite a normal LV ejection fraction. Whether severe secondary MR may be a specific therapeutic target in HFpEF patients deserves further study.

\section{References}

1 Maréchaux S, Pibarot P, Ennezat PV: Insights into functional mitral regurgitation despite preserved LVEF. Arch Cardiovasc Dis 2011; 104:131-133.

2 Maréchaux S, Neicu DV, Braun S, et al: Functional mitral regurgitation: a link to pulmonary hypertension in heart failure with preserved ejection fraction. J Card Fail 2011;17: 806-812.

Secondary Mitral Regurgitation in Heart Failure
3 Rossi A, Dini FL, Faggiano P, et al: Independent prognostic value of functional mitral regurgitation in patients with heart failure. A quantitative analysis of 1,256 patients with ischaemic and non-ischaemic dilated cardiomyopathy. Heart 2011;97:1675-1680.

4 Bursi F, Enriquez-Sarano M, Nkomo VT, et al: Heart failure and death after myocardial infarction in the community: the emerging role of mitral regurgitation. Circulation 2005; 111:295-301.
5 Hung J, Papakostas L, Tahta SA, et al: Mechanism of recurrent ischemic mitral regurgitation after annuloplasty: continued LV remodeling as a moving target. Circulation 2004; 110:1185-1190.

6 Grande-Allen KJ, Borowski AG, Troughton RW, et al: Apparently normal mitral valves in patients with heart failure demonstrate biochemical and structural derangements: an extracellular matrix and echocardiographic study. J Am Coll Cardiol 2005;45:54-61. 
7 Chaput M, Handschumacher MD, Guerrero JL, Holmvang G, Dal-Bianco JP, Sullivan S, Vlahakes GJ, Hung J, Levine RA, Leducq Foundation MITRAL Transatlantic Network: Mitral leaflet adaptation to ventricular remodeling: prospective changes in a model of ischemic mitral regurgitation. Circulation 2009;120:S99-S103.

8 Agricola E, Oppizzi M, Maisano F, et al: Echocardiographic classification of chronic ischemic mitral regurgitation caused by restricted motion according to tethering pattern. Eur J Echocardiogr 2004;5:326-334.

9 Magne J, Senechal M, Dumesnil JG, et al: Ischemic mitral regurgitation: a complex multifaceted disease. Cardiology 2009;112:244-259.

10 Lancellotti P, Moura L, Pierard LA, et al: European Association of Echocardiography recommendations for the assessment of valvular regurgitation. Part 2. Mitral and tricuspid regurgitation (native valve disease). Eur J Echocardiogr 2010;11:307-332.

11 Park S-M, Park SW, Casaclang-Verzosa G, et al: Diastolic dysfunction and left atrial enlargement as contributing factors to functional mitral regurgitation in dilated cardiomyopathy: data from the Acorn trial. Am Heart J 2009; 157:762.e3-e10.

12 Van de Veire NR, De Sutter J: Effect of ischemic mitral regurgitation on the ratio of early transmitral flow velocity to mitral annulus early diastolic velocity in patients with stable coronary artery disease. Am J Cardiol 2006; 97:1449-1451.

13 Maréchaux S, Pinçon C, Poueymidanette M, et al: Elevated left atrial pressure estimated by Doppler echocardiography is a key determinant of mitral valve tenting in functional mitral regurgitation. Heart 2010;96:289-297.

14 Enriquez-Sarano M, Rossi A, Seward JB, et al: Determinants of pulmonary hypertension in left ventricular dysfunction. J Am Coll Cardiol 1997;29:153-159.

15 Miller WL, Mahoney DW, Michelena HI, et al: Contribution of ventricular diastolic dysfunction to pulmonary hypertension complicating chronic systolic heart failure. JACC Cardiovasc Imaging 2011;4:946-954.

16 Keren G, Bier A, Strom JA, Laniado S, Sonnenblick EH, Le Jemtel TH: Dynamics of mitral regurgitation during nitroglycerin therapy: a Doppler echocardiographic study. Am Heart J 1986;112:517-525.

17 Seneviratne B, Moore GA, West PD: Effect of captopril on functional mitral regurgitation in dilated heart failure: a randomised double blind placebo controlled trial. Br Heart J 1994; 72:63-68.

18 Lowes BD, Gill EA, Abraham WT, Larrain JR, Robertson AD, Bristow MR, Gilbert EM: Effects of carvedilol on left ventricular mass, chamber geometry, and mitral regurgitation in chronic heart failure. Am J Cardiol 1999;83:1201-1205.

19 Yared K, Lam KM, Hung J: The use of exercise echocardiography in the evaluation of mitral regurgitation. Curr Cardiol Rev 2009;5:312322.
20 Lancellotti P, Lebrun F, Pierard LA: Determinants of exercise-induced changes in mitral regurgitation in patients with coronary artery disease and left ventricular dysfunction. J Am Coll Cardiol 2003;42:1921-1928.

21 Ennezat PV, Maréchaux S, Le Tourneau T, et al: Myocardial asynchronism is a determinant of changes in functional mitral regurgitation severity during dynamic exercise in patients with chronic heart failure due to severe left ventricular systolic dysfunction. Eur Heart J 2006;27:679-683.

22 Ennezat PV, Gal B, Kouakam C, et al: Cardiac resynchronisation therapy reduces functional mitral regurgitation during dynamic exercise in patients with chronic heart failure: an acute echocardiographic study. Heart 2006;92:10911095.

23 Maréchaux S, Pincon C, Gal B, et al: Functional mitral regurgitation at rest determines the acute hemodynamic response to cardiac resynchronization therapy during exercise: an acute exercise echocardiographic study. J Am Soc Echocardiogr 2009;22:464-471.

24 Breithardt O, Sinha A, Schwammenthal E, et al: Acute effects of cardiac resynchronization therapy on functional mitral regurgitation in advanced systolic heart failure. J Am College Cardiol 2003;41:765-770.

25 Lancellotti P, Gérard PL, Piérard LA: Longterm outcome of patients with heart failure and dynamic functional mitral regurgitation. Eur Heart J 2005;26:1528-1532.

26 Ennezat PV, Maréchaux S, Huerre C, et al: Exercise does not enhance the prognostic value of Doppler echocardiography in patients with left ventricular systolic dysfunction and functional mitral regurgitation at rest. Am Heart J 2008;155:752-757.

27 Piérard LA, Lancellotti P: The role of ischemic mitral regurgitation in the pathogenesis of acute pulmonary edema. N Engl J Med 2004; 351:1627-1634

28 Klapholz M, Maurer M, Lowe A, et al: Hospitalization for heart failure in the presence of a normal left ventricular ejection fraction: results of the New York Heart Failure Registry. J Am Coll Cardiol 2004;43:1432-1438.

29 Henkel DM, Redfield MM, Weston SA, et al: Death in heart failure: a community perspective. Circ Heart Fail 2008;1:91-97.

30 Massie BM, Carson PE, McMurray JJ, et al: Irbesartan in patients with heart failure and preserved ejection fraction. N Engl J Med 2008;359:2456-2467.

31 Yusuf S, Pfeffer MA, Swedberg K, et al: Effects of candesartan in patients with chronic heart failure and preserved left-ventricular ejection fraction: the CHARM-Preserved Trial. Lancet 2003;362:777-781.

32 Borlaug BA, Paulus WJ: Heart failure with preserved ejection fraction: pathophysiology, diagnosis, and treatment. Eur Heart J 2011;32: 670-679.
33 Ennezat PV, Maréchaux S, Six-Carpentier M, et al: Renal resistance index and its prognostic significance in patients with heart failure with preserved ejection fraction. Nephrol Dial Transplant 2011;26:3908-3913.

34 Maréchaux S, Six-Carpentier MM, Bouabdallaoui N, et al: Prognostic importance of comorbidities in heart failure with preserved left ventricular ejection fraction. Heart Vessels 2011;26:313-320.

35 Rusinaru D, Buiciuc O, Houpe D, et al: Renal function and long-term survival after hospital discharge in heart failure with preserved ejection fraction. Int J Cardiol 2011;147:278282.

36 Tribouilloy C, Rusinaru D, Mahjoub H, et al: Prognostic impact of diabetes mellitus in patients with heart failure and preserved ejection fraction: a prospective five-year study. Heart 2008;94:1450-1455.

37 Tribouilloy C, Rusinaru D, Mahjoub H, et al: Prognosis of heart failure with preserved ejection fraction: a 5 year prospective populationbased study. Eur Heart J 2008;29:339-347.

38 Butany J, Ahluwalia MS, Nair V, et al: Age-related morphological changes in cardiac valves. Geriatr Aging 2003;6:49-54

39 Barasch E, Gottdiener JS, Larsen EK, et al: Clinical significance of calcification of the fibrous skeleton of the heart and aortosclerosis in community dwelling elderly. The Cardiovascular Health Study (CHS). Am Heart J 2006;151:39-47.

40 Ennezat PV, Bellouin A, Maréchaux S, Juthier F, Fayad G, Vincentelli A, Berrébi A, Auffray JL, Bauchart JJ, Mouquet F, Montaigne D, Asseman P, Le Jemtel TH, Pibarot P: Pivotal role of bedside Doppler echocardiography in the assessment of patients with acute heart failure and mitral regurgitation. Cardiology 2009; 113:249-259.

41 Ennezat PV, Logeart D, Berrebi A, Vincentelli A, Maréchaux S: Key role of Doppler echocardiography in the emergency management of elderly patients. Arch Cardiovasc Dis 2010;103:115-128.

42 Melenovsky V, Borlaug BA, Rosen B, et al: Cardiovascular features of heart failure with preserved ejection fraction versus nonfailing hypertensive left ventricular hypertrophy in the urban Baltimore community: the role of atrial remodeling/dysfunction. J Am Coll Cardiol 2007;49:198-207.

43 Tanimoto M, Pai RG: Effect of isolated left atrial enlargement on mitral annular size and valve competence. Am J Cardiol 1996;77:769774 .

44 Gertz ZM, Raina A, Saghy L, et al: Evidence of atrial functional mitral regurgitation due to atrial fibrillation: reversal with arrhythmia control. J Am Coll Cardiol 2011;58:14741481.

45 Yu CM, Zhang Q, Yip GW, Lee PW, Kum LC, Lam YY, Fung JW: Diastolic and systolic asynchrony in patients with diastolic heart failure: a common but ignored condition. J Am Coll Cardiol 2007;49:97-105. 
46 Chang SA, Kim HK, Kim DH, et al: Left ventricular systolic and diastolic dyssynchrony in asymptomatic hypertensive patients. J Am Soc Echocardiogr 2009;22:337-342.

47 Park HE, Chang SA, Kim HK, et al: Impact of loading condition on the $2 \mathrm{D}$ speckle trackingderived left ventricular dyssynchrony index in nonischemic dilated cardiomyopathy. Circ Cardiovasc Imaging 2010;3:272-281.

48 Hung J, Otsuji Y, Handschumacher MD, et al: Mechanism of dynamic regurgitant orifice area variation in functional mitral regurgitation: physiologic insights from the proximal flow convergence technique. J Am Coll Cardiol 1999;33:538-545.

49 Agricola E, D'Amato R, Stella S, et al: Effects of mild ischemic mitral regurgitation on ventricular remodeling and its contribution to congestive heart failure. J Am Soc Echocardiogr 2011;24:1376-1382.

50 Holtz JE, Upadhyaya DS, Cohen BE, et al: $\mathrm{Mi}$ tral annular calcium, inducible myocardial ischemia, and cardiovascular events in outpatients with coronary heart disease (from the Heart and Soul Study). Am J Cardiol 2012; 109:1092-1096.
51 Lam CS, Roger VL, Rodeheffer RJ, et al: Pulmonary hypertension in heart failure with preserved ejection fraction: a community-based study. J Am Coll Cardiol 2009;53:1119-1126.

52 Maréchaux S, Terrade J, Biausque F, et al: Exercise-induced functional mitral regurgitation in heart failure and preserved ejection fraction: a new entity. Eur J Echocardiogr 2010; 11:E14.

53 Ennezat PV, Lefetz Y, Maréchaux S, et al: Left ventricular abnormal response during dynamic exercise in patients with heart failure and preserved left ventricular ejection fraction at rest. J Card Fail 2008;14:475-480.

54 Pecini R, Hammer-Hansen S, Dalsgaard M, et al: Determinants of exercise-induced increase of mitral regurgitation in patients with acute coronary syndromes. Echocardiography 2010; 27:567-574.

55 Bouzas-Mosquera A, Peteiro J: Mitral regurgitation during exercise in patients with left ventricular systolic dysfunction. Am Heart J 2008; 156:e27.

56 Ha JW, Oh JK, Pellikka PA, et al: Diastolic stress echocardiography: a novel noninvasive diagnostic test for diastolic dysfunction using supine bicycle exercise Doppler echocardiography. J Am Soc Echocardiogr 2005;18:63-68.
57 Tan YT, Wenzelburger F, Lee E, et al: The pathophysiology of heart failure with normal ejection fraction: exercise echocardiography reveals complex abnormalities of both systolic and diastolic ventricular function involving torsion, untwist, and longitudinal motion. J Am Coll Cardiol 2009;54:36-46.

58 Borlaug BA, Olson TP, Lam CS, et al: Global cardiovascular reserve dysfunction in heart failure with preserved ejection fraction. J Am Coll Cardiol 2010;56:845-854.

59 Maeder MT, Thompson BR, Brunner-La Rocca HP, et al: Hemodynamic basis of exercise limitation in patients with heart failure and normal ejection fraction. J Am Coll Cardiol 2010;56:855-863.

60 Donal E, Thebault C, Lund LH, et al: Heart failure with a preserved ejection fraction additive value of an exercise stress echocardiography. Eur Heart J Cardiovasc Imaging 2012; 13:656-665.

61 Donal E, Lund LH, Linde C, et al: Rationale and design of the Karolinska-Rennes (KaRen) prospective study of dyssynchrony in heart failure with preserved ejection fraction. Eur J Heart Fail 2009;11:198-204. 